\title{
Analysis of oxygen binding by hemoglobin on the basis of mean intrinsic thermodynamic quantities
}

\author{
Abdol-Khalegh Bordbar ${ }^{\bowtie}$, Sayed Habib-Allah Mousavi and Hamid Dazhampanah \\ Laboratory of Biophysical Chemistry, Department of Chemistry, Isfahan University, Isfahan, Iran; \\ ®e-mails: bordbar@chem.ui.ac.ir and khalegh_bordbar@yahoo.com
}

Received: 01 May, 2006; revised: 18 August, 2006; accepted: 04 September, 2006 available on-line: 01 October, 2006

\begin{abstract}
The binding data for oxygenation of human hemoglobin, $\mathrm{Hb}$, at various temperatures and in the absence and presence of 2,3-diphosphoglycerate, DPG, and inositol hexakis phosphate, IHP, were analyzed for extraction of mean intrinsic Gibbs free energy, $\overline{\Delta G}^{\mathrm{o}}$, enthalpy, $\overline{\Delta H}^{\mathrm{o}}$, and entropy, $\overline{\Delta S}^{\circ}$, of binding at various partial oxygen pressures. This method of analysis considers all the protein species present such as dimer and tetramer forms which were not considered by Imai et al. (Imai K et al., 1970, Biochim Biophys Acta 200: 189-196), in their analysis which was based on Adair equation. In this regard, the values of Hill equation parameters were estimated with high precision at all points of the binding curve and used for calculation of $\overline{\Delta G}^{\mathrm{o}}, \overline{\Delta H}^{\mathrm{o}}$ and $\overline{\Delta S}^{\mathrm{o}}$ were also calculated by analysis of $\overline{\Delta G}^{0}$ values at various temperatures using van't Hoff equation. The results represent the enthalpic nature of the cooperativity in $\mathrm{Hb}$ oxygenation and the compensation effect of intrinsic entropy. The interpretation of results also to be, into account the decrease of the binding affinity of sites for oxygen in the presence of DPG and IHP without any considerable changes in the site-site interaction (extent of cooperativity). In other words, the interactions between bound ligands, organic phosphates and oxygen, are more due to a decreasing binding affinity and not to the reduction of the cooperative interaction between sites. The results also document the more heterotropic effect of IHP compared to DPG.
\end{abstract}

Keywords: hemoglobin, cooperativity, mean intrinsic Gibbs free energy, linkage phenomena

\section{INTRODUCTION}

Hemoglobin, $\mathrm{Hb}$, is the respiratory protein in red blood cells of vertebrates. Its physiological functions are transport of oxygen from lungs to tissues and transfer of carbon dioxide from tissues to lungs. The equilibrium of oxygen binding is influenced by organic phosphates such as 2,3-diphosphoglycerate, DPG, which is present in red blood cells of mammals and inositol hexakis phosphate, IHP, which is related to inositol pentakis phosphate found in red blood cells of birds (Baldvin, 1975). This organic phosphate has heterotropic allostric effect on oxygenation of hemoglobin. Some hemoglobin properties in this regard are interpreted on the basis of fully liganded and/or unliganded hemoglobin structures, while other its properties are explained on the basis of intermediate structures and alternative oligomeric forms of hemoglobin. These are not directly visible and must be illustrate by a model (Roughton, 1936; Imai \& Yonetani, 1974; 1975; Imai, 1979; Yamamoto \& Nagaoka 1998; Scalonia et al., 1999; Labergea et al., 2005).

The shape of oxygen equilibrium curves is invariant for temperature changes and this led Wyman (1948) to conclude that the heat of oxygenation is the same for all the hemes, and the cooperative effects are essentially entropic in nature. In contrast Imai and Yonetani (1975) believe that the cooperativity of hemoglobin oxygenation may be due to both enthalpic and entropic origins. Imai and Yonetani analyzed the oxygen-hemoglobin binding data by fitting them to Adair equation (Adair, 1925; Adair et al., 
1925; Baldvin, 1975; Imai \& Yonetani, 1975). While the estimation of sequential binding constants is possible by this method, the accuracy of the calculated parameters is strongly dependent on the estimation of the initial values and fitting procedures. For example, Brodersen et al. $(1987$; 1988) reported 30 different acceptable solutions for the oxygen$\mathrm{Hb}$ system. Thus, there is a significant uncertainty on the interpretations that arise from these fitting data. Moreover, those authors did not consider the presence of the dimer form of hemoglobin in their analyzing method. Besides these sequential binding values, the estimation of mean binding parameters is also possible for this system. While it seems that these mean parameters may be less informative than the sequential ones, their estimation is more precise.

In the present work, the mean intrinsic Gibbs free energy, $\overline{\Delta G}^{\circ}$, enthalpy, $\overline{\Delta H}^{\circ}$ and entropy, $\overline{\Delta S}^{\circ}$, of human hemoglobin oxygenation at various experimental conditions such as temperature, presence and absence of DPG and IHP, were calculated using modified form of a formula which was derived earlier (Bordbar et al., 1997). Some new features of the oxygen $-\mathrm{Hb}$ equilibrium such as the enthalpic or entropic origin of cooperativity and the nature of the allostric effects of DPG and IHP were revealed through this new analyzing method.

\section{DATA ANALYSIS}

The oxygen- $\mathrm{Hb}$ binding data were taken directly from literature (Imai \& Yonetani, 1974). These data were determined by an automatic recording method as described elsewhere (Imai et al., 1970). The buffers used were $0.05 \mathrm{M}$ bis-Tris with and without $2 \mathrm{mM}$ DPG and $2 \mathrm{mM}$ IHP, and contained $0.1 \mathrm{M} \mathrm{Cl}^{-}$which was adjusted with sodium chloride. The $\mathrm{pH}$ of the buffers was adjusted to 7.4 at the same temperatures as for oxygen equilibrium measurements, i.e. at $10,15,20,25,30$ and $35^{\circ} \mathrm{C}$.

The corresponding Hill plots were constructed as the variation of $\log (\mathrm{Y} /(1-\mathrm{Y}))$ versus $\log P_{\mathrm{O}^{\prime}}$, where $\mathrm{Y}$ and $P_{\mathrm{O}_{2}}$ are fractional saturation of $\mathrm{Hb}$ with oxygen and partial oxygen pressure, respectively. The Hill plots were fitted to a suitable equation using Sigma Plot software (http://www.spss.com/software/ science/sigmaplot/, and for more information refer to http://www.spssscience.com/). This fitting equation interpolated the experimental data with high precision and was used for determination of exact values of Hill coefficient, $n_{\mathrm{H}^{\prime}}$ and Hill binding constant, $K_{\mathrm{H}^{\prime}}$ at any specified value of $P_{\mathrm{O}_{2}}$. These parameters were calculated using Eqns. (1) and (2), respectively (Hill, 1910)

$n_{H}=\frac{d \ln (Y / 1-Y)}{d \ln \left(P_{O_{2}} / P^{o}\right)}$
$K_{H}=\frac{(Y / 1-Y)^{1 / n_{H}}}{P_{O_{2}}}$

where $P^{\mathrm{o}}$ is pressure of gas at standard conditions of 1 bar. The unit of $K_{\mathrm{H}}$ is torr ${ }^{-1}$. The fitted Hill plots and the variation of calculated $n_{\mathrm{H}}$ versus $\log P_{\mathrm{O}_{2}}$ at various temperatures and in the absence or presence of DPG and IHP are shown in Figs. 1 and 2, respectively.

The following multiple equilibria must exist in solution:

$\mathrm{Hb}^{\mathrm{D}}+\mathrm{O}_{2} \stackrel{\mathrm{K}_{1}^{\mathrm{eD}}}{\longrightarrow} \mathrm{Hb}^{\mathrm{D}} \mathrm{O}_{2}$

$\mathrm{Hb}^{\mathrm{D}} \mathrm{O}_{2}+\mathrm{O}_{2} \stackrel{\mathrm{K}_{2}^{\mathrm{dD}}}{\longrightarrow} \mathrm{Hb}^{\mathrm{D}}\left(\mathrm{O}_{2}\right)_{2}$

and

$\mathrm{Hb}^{\mathrm{T}}+\mathrm{O}_{2} \stackrel{\mathrm{K}_{1}^{\mathrm{oT}}}{\longrightarrow} \mathrm{Hb}^{\mathrm{T}} \mathrm{O}_{2}$

$\mathrm{Hb}^{\mathrm{T}} \mathrm{O}_{2}+\mathrm{O}_{2} \stackrel{\mathrm{K}_{2}^{0 \mathrm{~T}}}{\longrightarrow} \mathrm{Hb}^{\mathrm{T}}\left(\mathrm{O}_{2}\right)_{2}$

$\mathrm{Hb}^{\mathrm{T}}\left(\mathrm{O}_{2}\right)_{2}+\mathrm{O}_{2} \stackrel{\mathrm{K}_{3}^{6 \mathrm{~T}}}{\longrightarrow} \mathrm{Hb}^{\mathrm{T}}\left(\mathrm{O}_{2}\right)_{3}$

$\mathrm{Hb}^{\mathrm{T}}\left(\mathrm{O}_{2}\right)_{3}+\mathrm{O}_{2} \stackrel{\mathrm{K}_{4}^{\mathrm{oT}}}{\longrightarrow} \mathrm{Hb}^{\mathrm{T}}\left(\mathrm{O}_{2}\right)_{4}$
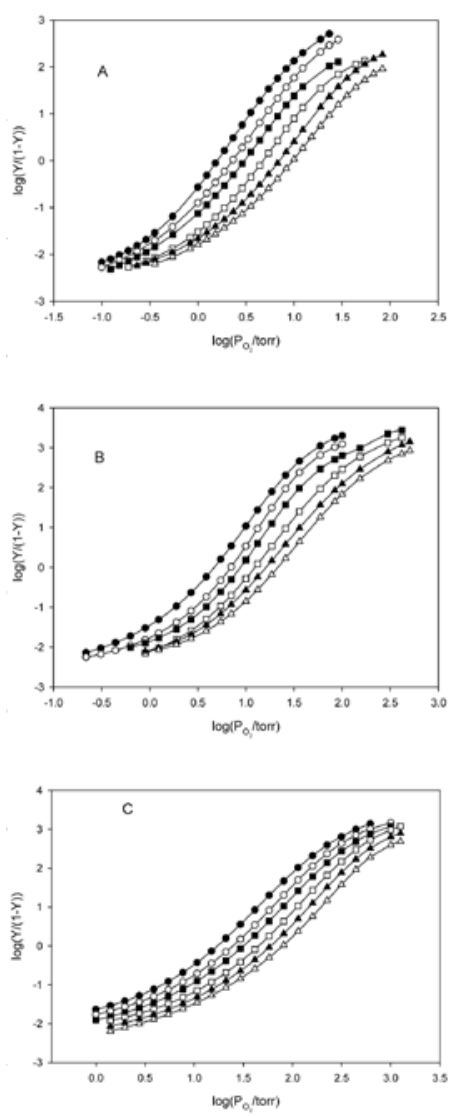

Figure 1. Hill plots of oxygen binding by $\mathrm{Hb}$.

Heme concentration, $60 \mu \mathrm{M}$; $\mathrm{pH} 7.4$, in $0.05 \mathrm{M}$ bis-Tris buffers containing $0.1 \mathrm{M} \mathrm{Cl}^{-},(\mathrm{A})$ in the absence of organic phosphate, (B) in the presence of $2 \mathrm{mM}$ DPG, (C) in the

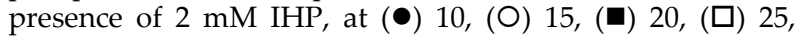
(ム) 30 , and $(\triangle) 35^{\circ} \mathrm{C}$. Continuous lines correspond to the best fitting equation. 
where $K^{\mathrm{oD}}$ and $K^{\mathrm{oT}}{ }_{j}$ are stepwise intrinsic binding constants for binding of $\mathrm{jth} \mathrm{O}_{2}$ to dimer, $\mathrm{Hb}^{\mathrm{D}}$, and tetramer, $\mathrm{Hb}^{\mathrm{T}}$, forms of $\mathrm{Hb}$, respectively. The intrinsic binding free energy corresponding to these binding constants should be equal to $\Delta G^{\mathrm{OD}}{ }_{\mathrm{j}}=-R T \ln \left(K^{\mathrm{oD}}{ }_{\mathrm{j}}\right.$ $\left.\times P^{\mathrm{o}}\right)$ and $\Delta G^{\mathrm{oT}}{ }_{\mathrm{j}}=-R T \ln \left(K^{\mathrm{OT}}{ }_{\mathrm{j}} \times P^{\mathrm{o})}\right.$, respectively.

The mean intrinsic Gibbs free energy per mole of monomer at any specified value of $P_{\mathrm{O}_{2}}, \overline{\Delta G^{\circ}}$, is defined as:

$$
\overline{\Delta G^{o}}=(1 / 4) \sum_{i=1}^{4} \sum_{j=1}^{i} x_{i}^{T} \Delta G_{j}^{o T}+(1 / 2) \sum_{i=1}^{2} \sum_{j=1}^{i} x_{i}^{D} \Delta G_{j}^{o D}
$$

where $x^{\mathrm{T}}{ }_{\mathrm{i}}$ and $x^{\mathrm{D}}{ }_{\mathrm{i}}$ are mole fractions of $\mathrm{Hb}^{\mathrm{T}}\left(\mathrm{O}_{2}\right)_{\mathrm{i}}$ and $\mathrm{Hb}^{\mathrm{D}}\left(\mathrm{O}_{2}\right)_{\mathrm{i}^{\prime}}$, respectively. $\overline{\Delta G}^{\mathrm{o}}$ can be calculated using the following equation:

$$
\overline{\Delta G^{o}}=-n_{H} R T \ln \left(K_{H} \times P^{o}\right)+\left(1-n_{H}\right) R T \ln \left(P_{O_{2}} / P^{o}\right)
$$

where $\mathrm{R}$ and $\mathrm{T}$ are universal gas constant and absolute temperature, respectively. This equation is a modified form of the equation which was previously obtained by Bordbar et al. (1997) and applied for binding of ionic surfactants to globular proteins. For
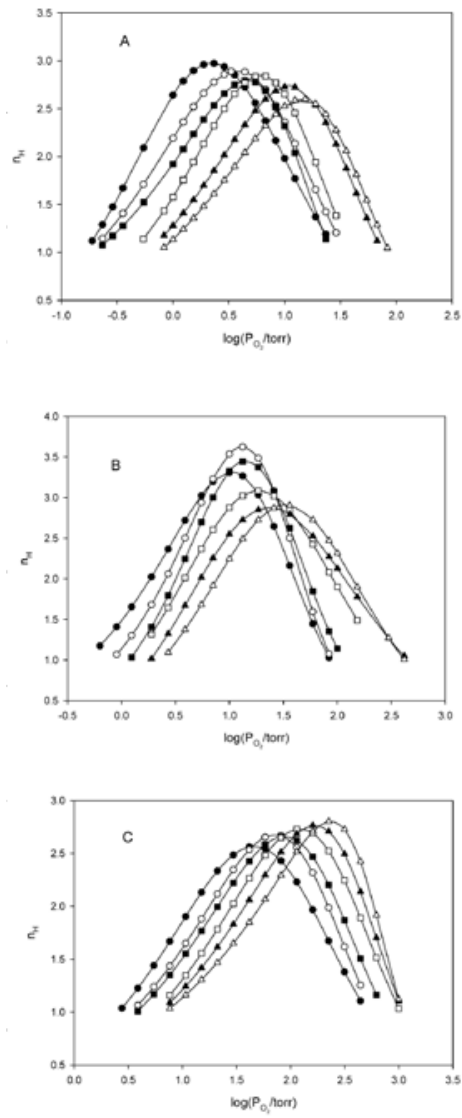

Figure 2. Variation of Hill coefficient, $n_{\mathrm{H}^{\prime}}$ versus $\log P_{\mathrm{O}_{2}}$. Oxygenation of $\mathrm{Hb}$ in the absence of organic phosphates (A), presence of $2 \mathrm{mM} \mathrm{DPG} \mathrm{(B),} \mathrm{and} \mathrm{presence} \mathrm{of} 2 \mathrm{mM}$

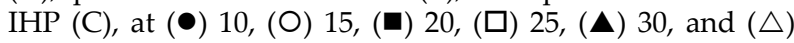
$35{ }^{\circ} \mathrm{C}$. The $n_{\mathrm{H}}$ values at any specified point were calculated by derivation of fitting equation of Hill plots (Fig. 1).
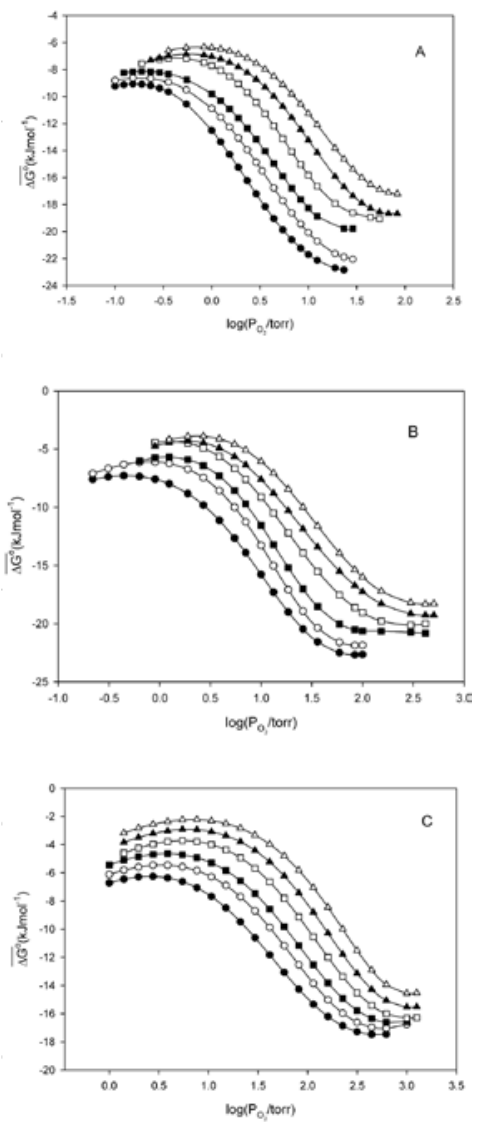

Figure 3. Variation of mean intrinsic Gibbs free energy, $\overline{\Delta G}^{0}$, versus $\log P_{\mathrm{O}}$

Oxygenation of $\mathrm{HB}^{\circ}$ in the absence of organic phosphate (A), presence of $2 \mathrm{mM}$ DPG (B), and presence of $2 \mathrm{mM}$

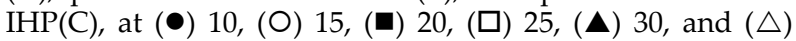
$35^{\circ} \mathrm{C}$.

the modification of the Bordbar equation, the existence of a single set of binding sites in $\mathrm{Hb}$ and the variable nature of $n_{\mathrm{H}}$ and $K_{\mathrm{H}^{\prime}}$ were considered. In order to dimensionless of logarithm arguments, the $K_{\mathrm{H}}$ multiply to $P^{\circ}$ in the first term and $P_{\mathrm{O}_{2}}$ divided to $P^{\circ}$ in the second term of Eqn. (4).

Figure 3 shows the variation of $\overline{\Delta G}^{\mathrm{o}}$ versus $\log P_{\mathrm{O}_{2}}$ for oxygenation of $\mathrm{Hb}$ in the absence and presence of DPG and IHP, respectively. The variation of $\overline{\Delta G}^{\circ}$ is also shown in Fig. 4 , at $25^{\circ} \mathrm{C}$ in the ab-

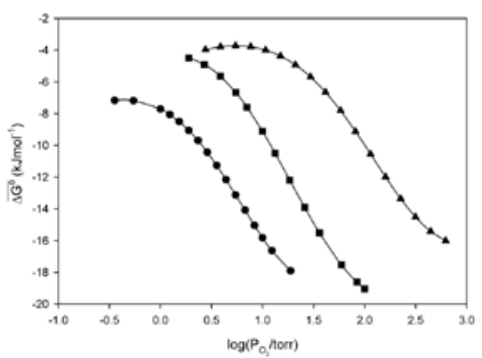

Figure 4. Variation of mean intrinsic Gibbs free energy $\overline{\Delta G}^{\mathrm{o}}$, versus $\log P_{\mathrm{O}}$.

Oxygenation of $\mathrm{Hb}^{2}$ at $25^{\circ} \mathrm{C}$ in the absence of organic phosphate $(\bullet)$, presence of $2 \mathrm{mM} \mathrm{DPG}(\boldsymbol{\square})$ and presence of 2 $\mathrm{mM} \operatorname{IHP}(\boldsymbol{\Delta})$. 

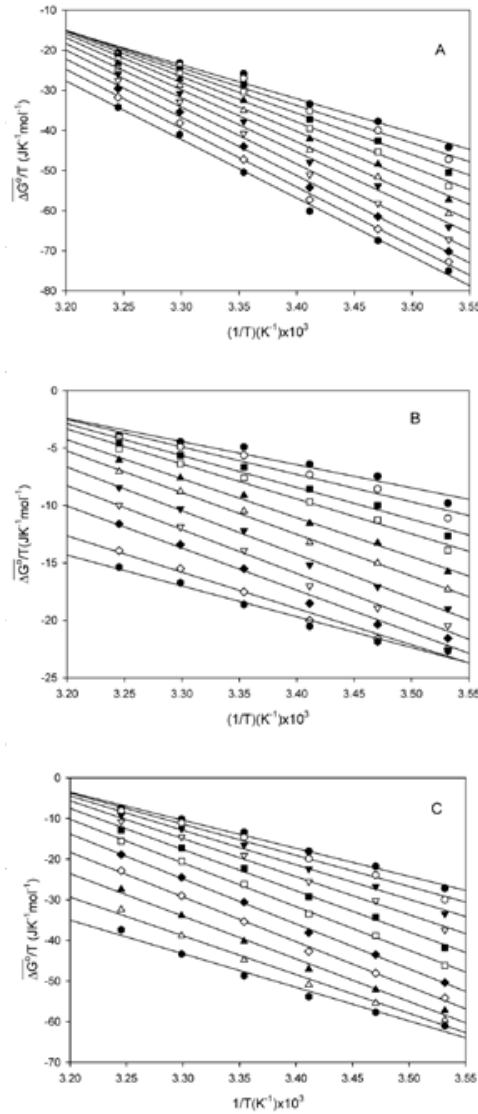

Figure 5. Variation of $\overline{\Delta G}^{0} / T$ versus $1 / T$ for oxygenation of $\mathbf{H b}$ :

(A) in the absence of organic phosphate, $(\bullet) P_{O_{2}}=1,(\mathrm{O})$ $P_{\mathrm{O}_{2}}=1.236$, (口) $P_{\mathrm{O}_{2}}=1.528$, (口) $P_{\mathrm{O}_{2}}=1.888,(\boldsymbol{\Delta}) P_{\mathrm{O}_{2}}=$ 2.333, $(\triangle) P_{\mathrm{O}_{2}}=2.884,(\nabla) P_{\mathrm{O}_{2}}=3.565,(\nabla) P_{\mathrm{O}_{2}}=4.395,(\nabla)$ $P_{\mathrm{O}_{2}}=5.445,(\diamond) P_{\mathrm{O}_{2}}=6.730,(-) P_{\mathrm{O}_{2}}=8.318$ torr, $(\mathrm{B})$ in the presence of $2 \mathrm{mM}$ DPG, $(\bullet) P_{O_{2}}=2.698$, (O) $P_{O_{2}}=3.873$, (ロ) $P_{\mathrm{O}_{2}}=5.483$, (口) $P_{\mathrm{O}_{2}}=7.047,(\boldsymbol{\Delta}) P_{\mathrm{O}_{2}}=10.000,(\triangle) P_{\mathrm{O}_{2}}=$ $13.274,(\nabla) P_{\mathrm{O}_{2}}=18.535$, ( $) P_{\mathrm{O}_{2}}=25.882,(\diamond) P_{\mathrm{O}_{2}}=36.141$, $(\diamond) P_{O_{2}}=59.293$, ( ) $P_{O_{2}}=83.946$, and $(C)$ in the presence of $2 \mathrm{mM} \mathrm{IHP},(\bullet) P_{\mathrm{O}_{2}}=10.7,(\mathrm{O}) P_{\mathrm{O}_{2}}=14.8,(\boldsymbol{\nabla}) P_{\mathrm{O}_{2}}=21.0$,

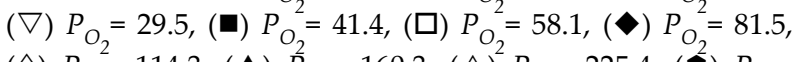
$(\diamond) P_{\mathrm{O}_{2}}=114.3,(\boldsymbol{\Delta}) P_{\mathrm{O}_{2}}=160.3,(\triangle)^{2} P_{\mathrm{O}_{2}}=225.4,(\boldsymbol{\bullet}) P_{\mathrm{O}_{2}}=$
315.5 torr.

sence and presence of DPG and IHP. The van't Hoff plots, $\overline{\Delta G}^{\circ} / T$ versus $1 / T$, are shown in Fig. 5 . With respect to Eqn. (5) the slope and y-intercept of this plot are equal to the mean intrinsic heat change $\overline{\Delta H}^{\mathrm{o}}$, and the negative value of the mean intrinsic entropy change, $-\overline{\Delta S}$ at any specific value of $P_{O_{2}}$, respectively. The variation of $\overline{\Delta H}^{\circ}$ and $\overline{T \Delta S}^{\circ}$ versus $\log P_{\mathrm{O}_{2}}$ are shown in Fig. 6.

$\overline{\Delta G}^{\mathrm{o}} / \mathrm{T}=\overline{\Delta H}^{\mathrm{o}} / \mathrm{T}-\overline{\Delta S}^{\mathrm{o}}$

\section{RESULTS AND DISCUSSION}

The oxygenation data and curve fitting are presented as the Hill plots in Fig. 1, where the ob-

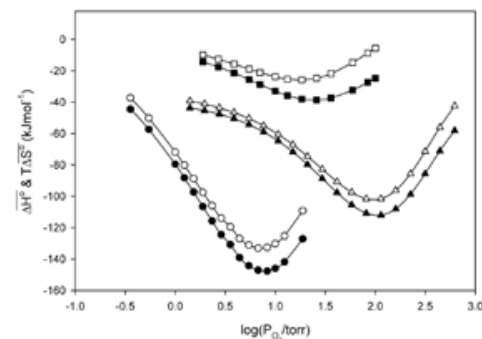

Figure 6. Variation of $\overline{\Delta H^{\circ}}(\bullet, \square$ and $\Delta)$ and $\overline{\mathrm{T} \Delta S^{\circ}}(\bigcirc$, and $\triangle$ ) versus $P_{\mathrm{O}}$.

Oxygenation of $\mathrm{Hb}$, in the absence of organic phosphate (circles), in the presence of $2 \mathrm{mM}$ DPG (rectangles) and in the presence of $2 \mathrm{mM} \mathrm{IHP}$ (triangles).

served points are compared with calculated lines. They show excellent fit along the saturation that was covered by experiments. It is evident that the position of the oxygen equilibrium curve depends on the temperature and the presence of DPG or IHP. It also shows that the lower asymptotes converge, while the upper asymptotes diverge, indicating that the initial steps of hemoglobin oxygenation depend on the temperature less markedly than the final steps.

As seen in Fig. 2, the maximum value of Hill coefficient, $n_{\mathrm{H} \text {,max }}$ also depends on the temperature; it become smaller as the temperature is going up in the absence of allosteric ligands (DPG and IHP) and in the presence of DPG. However, it slightly increases with increasing temperature in the presence of IHP.

The mean intrinsic Gibbs free energy is shifted to the right and to less negative values, with increasing temperature (Fig. 3). This means that increasing the temperature reduces both the intrinsic binding affinity of the sites and the cooperative interactions between them. Figure 4 shows the variation of $\overline{\Delta G}^{\circ}$ versus $\log P_{O}$ at $25^{\circ} \mathrm{C}$ in the absence and presence of DPG and IHP. It is obvious that the effect of DPG and IHP on the binding site affinity is stronger than that on the cooperative interaction between the sites.

The linearity of van't Hoff plots, in the absence and presence of a ligand (Fig. 5), expressing the null value of heat capacity change of heme oxy-

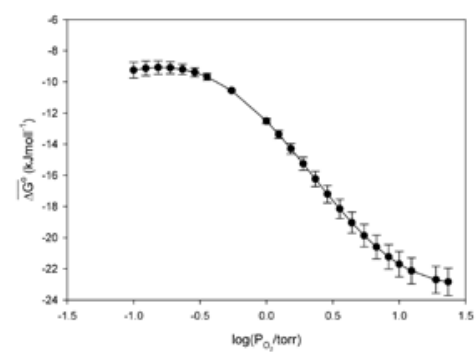

Figure 7. Variation of mean intrinsic Gibbs free energy, $\bar{\Delta} G^{\mathbf{o}}$, versus $\log P_{\mathrm{O}}$

Oxygenation of $\mathrm{Hb}^{2}$ in the absence of organic phosphate at $10^{\circ} \mathrm{C}$. The error bars were constructed by considering $5 \%$ relative error in estimated values of $n_{\mathrm{H}}$ and $K_{\mathrm{H}}$. 
genation, $\Delta C_{\mathrm{p}}^{\mathrm{o}}$. All enthalpy values correspond to association reactions of $\mathrm{O}_{2}$ with $\mathrm{Hb}$, are negative at various temperatures and pressures (Fig. 6). The amount of heat released is increased as the oxygen pressure becomes higher. The heat released of $\mathrm{Hb}$ oxygenation is markedly decreased in the presence of DPG and IHP. This can be due to significant contributions of the various solute species in the heat of oxygenation through their oxygen-linked binding to hemoglobin. The released heat of oxygenation increases as $P_{\mathrm{O}_{2}}$ goes up. So, the first steps of hemoglobin oxygenation are influenced more than the last steps by these factors, this is also confirmed by the fact that the intrinsic equilibrium constant for the last step, $\mathrm{K}^{\mathrm{o}}{ }_{4}$ is insensitive to $\mathrm{pH}$ changes and the presence of $\mathrm{Cl}^{-}$, DPG and IHP, while $\mathrm{K}_{1}^{\mathrm{o}}, \mathrm{K}^{\mathrm{o}}{ }_{2}$, and $\mathrm{K}_{3}^{\mathrm{O}}$ are markedly influenced by these factors (Imai 1973, 1974; Tyuma et al., 1973; Atha \& Ackers 1974). The results mean that a great majority of DPG and IHP bound to deoxyhemoglobin are released prior to the 4 th oxygenation step. On the basis of the difference released heat in the absence and presence of DPG (30 to $109 \mathrm{~kJ} \cdot \mathrm{mol}^{-1}$ ) and IHP (1 to $36 \mathrm{~kJ} \cdot \mathrm{mol}^{-}$ $\left.{ }^{1}\right)$, the association or dissociation of DPG and IHP ions by hemoglobin is accomplished by exchange a significant amount of heat. However, the final steps are more exothermic than the initial ones.

The behavior of the mean intrinsic entropy of hemoglobin oxygenation, $\overline{\Delta S}^{\circ}$, is similar to that of $\overline{\Delta H}^{\circ}$, in the absence or presence of DPG and IHP. This indicates the existence of an enthalpy-entropy compensation phenomenon (Fig. 6). The absolute value of entropy is reduced in the presence of DPG $\left(92\right.$ to $\left.359 \mathrm{JK}^{-1} \cdot \mathrm{mol}^{-1}\right)$ and IHP $\left(6\right.$ to $\left.105 \mathrm{JK}^{-1} \cdot \mathrm{mol}^{-1}\right)$, while their values are still negative. The negative value of entropy is under the noticeable in light of the associative nature of the reaction. The increasing entropy in the presence of DPG or IHP can be related to the release of various ligands during hemoglobin oxygenation.

The cooperativity effect can be considered by comparison of $\overline{\Delta H}^{\circ}$ with $\overline{T \Delta S^{\circ}}$ at a specified temperature, i.e. $25^{\circ} \mathrm{C}$ (Fig. 4). Negative values of enthalpy have a positive effect on the cooperativity, whereas negative values of entropy have an opposite effect. In the absence of DPG and IHP, this compensation shows that the changes of $\overline{\Delta H}^{\circ}$ and $\overline{T \Delta S}^{\circ}$ have the same fashion, and their difference is small (5 to 15 $\left.\mathrm{kJ} \cdot \mathrm{mol}^{-1}\right)$. In the presence of organic phosphates, the changes of $\overline{\Delta H}^{\circ}$ and $\overline{T \Delta S}^{\circ}$ are similar and their difference is in the same value range as in the absence of organic phosphate, in other words, the reduction in the values of entropy and enthalpy in the presence and absence of organic phosphate are approximately equal. Hence the presence of organic phosphates has no affects on the derivative of mean intrinsic Gibbs free energy (Fig. 3), and just causes a shift of Gibbs free energy curve to the right. This means that the presence of organic phosphate just affects the intrinsic binding affinity of the sites for oxygen, and does not influence the cooperative interaction between them.

\section{CONCLUSION}

An analysis of oxygen binding data on the basis of their fitting to Adair equation has been done previously and most of the interpretations presented are based on the results of this method of analysis (Imai \& Yonetani, 1974; 1975; Imai, 1979). The major shortcomings of this method are the limitations of this equation in covering all data; the calculated results are of low precision and accuracy, and dimer contribution is neglected. In this work, we fit the binding data to a suitable equation with high precision that subsequently allowed us to calculate Hill binding parameters with a thermodynamic basis through Eqn. (4). Thus, our results are more precisely analyzed for extraction of mean intrinsic thermodynamic quantities. We believe that this new analysis method can be also used for any binding system and its importance is going up with an increasing number of binding sites which creates more uncertainty in the estimated values of Adair binding constants.

The entropic origin of the cooperativity of hemoglobin oxygenation was introduced by Wyman (1948), whereas Imai and Yonetani (1975) believe that the cooperativity may have both enthalpic and entropic origins. With respect to the negative values of mean intrinsic enthalpy and entropy of hemoglobin oxygenation in the absence and presence of organic phosphates (Fig. 6), enthalpy causes a decrease of the mean intrinsic Gibbs free energy, while entropy increases it. Therefore, we propose that the cooperativity of hemoglobin oxygenation has enthalpic origin and entropic compensation. The interpretation of the results shows a decrease of the site binding affinity in the presence of organic phosphate without any considerable changes in the amount of site-site interaction (extent of cooperativity). On the other hand, the heterotropic linkage phenomenon in simultaneous binding of organic phosphate and oxygen to $\mathrm{Hb}$ is more due to decreasing of binding affinity of sites and less due to reducing of cooperative interaction between sites.

The sensitivity of this approach to the uncertainty in Hill parameters was also tested and the result is shown in Fig. 7. This figure shows the variation of calculated $\overline{\Delta G}^{\circ}$ versus $\log P_{\mathrm{O}_{2}}$ at $10^{\circ} \mathrm{C}$ and in the absence of DPG and IHP, by considering 5\% relative error in estimated $n_{\mathrm{H}}$ and $K_{\mathrm{H}}$ values. The relative error in $\overline{\Delta G}^{\circ}$ was less than $5 \%$ in all points. 
Moreover, the trend of variation of $\overline{\Delta G}^{\circ}$ that has an essential role in our interpretation, shows little dependency on such errors. This shows that the proposed method is more reliable compared to Adair equation fitting.

Several blood diseases are due to structural and genetic defects of hemoglobin (Chinchanga et al., 2005; Nishino et al., 2006; Shmukler et al., 2000; Stathopulos, 2003; Steiper et al., 2006). These diseases could affect hemoglobin oxygenation activity. Our approach can be used to investigate the effect of these structural changes on function of $\mathrm{Hb}$. The obtained results may lead us to designing new drugs for therapy of these diseases.

\section{REFERENCES}

Adair GS (1925) The hemoglobin system VI. The oxygen dissociation curve of hemoglobin. J Biol Chem 63: 529545.

Adair GS, Bock AV, Field H Jr (1925) The hemoglobin system I. Classification of reactions. J Biol Chem 63: 493497.

Atha DH, Ackers GK (1974) Human hemoglobin as a function of $\mathrm{pH}$ and the extent of reaction. Biochemistry 13: 2376-2382.

Baldvin JM (1975) Structure and function of haemoglobin. In: Progress in Biophysics and Molecular Biology, vol 29, pp 225-320, Pergamon Press, Great Britain.

Bordbar AK, Sabory AA, Housaindokht MR, Moosavi Movahedi AA (1997) Statistical effects of the binding of ionic surfactant to protein. J Coll Int Sci 192: 415-419.

Brodersen R, Nielsen F, Christian JC, Andersen K (1987) Characterization of binding equilibrium data by a variety of fitted isotherms. Eur J Biochem 169: 487-495.

Brodersen R, Honore B, Pedersen AO, Klotz IM (1988) Binding constants for ligand-carrier complexes. Trends Pharmacol Sci 9: 252-257.

Chinchang W, Viprakasit V, Pung-Amritt P, Tanphaichitr VS, Yenchitsomanusb P (2005) Molecular analysis of unknown $\beta$-globin gene mutations using polymerase chain reaction-single strand conformation polymorphism (PCR-SSCP) technique and its application in Thai families with $\beta$-thalassemias and $\beta$-globin variants. Clin Biochem 38: 987-996.

Hill, A.V. (1910) The heat produced by contracture and muscular tone. J Physiol 40: 389-403.

Imai K (1973) Analyses of oxygen equilibria of native and chemically modified human adult hemoglobins on the basis of Adair's stepwise oxygenation theory and the allosteric model of Monod, Wyman, and Changeux. Biochemistry 12: 798-808.
Imai K (1974) Hemoglobin Chesapeaks (92 $\alpha$, arginine leucine). Precise measurements and analysis of oxygen equilibrium. J Biol Chem 249: 7607-7612.

Imai K (1979) Thermodynamic aspects of the co-operativity in four-step oxygenation equilibria of haemoglobin. J Mol Biol 133: 233-247.

Imai K, Yonetani $\mathrm{T}$ (1974) $\mathrm{pH}$ Dependence of the Adair constants of human hemoglobin. J Biol Chem 250: 22272231.

Imai K, Yonetani T (1975) Thermodynamical studies of oxygen equilibrium of hemoglobin. J Biol Chem 250: 7093-7098.

Imai $\mathrm{K}$, Morimoto $\mathrm{H}$, Kotani $\mathrm{M}$, Watari $\mathrm{H}$, Hirata W, Kuroda M (1970) Studies on the function of abnormal hemoglobins I. An improved method for automatic measurement of the oxygen equilibrium curve of hemoglobin. Biochim Biophys Acta 200: 189-196.

Labergea M, Kövesia I, Yonetani T, Fidya J (2005) R-state hemoglobin bound to heterotropic effectors: models of the DPG, IHP and RSR13 binding sites. FEBS Lett 579: 627-632.

Nishino T, Tubb J, Emery DW (2006) Partial correction of murine $\beta$-thalassemia with a gammaretrovirus vector for human $\gamma$-globin. Blood Cells Mol Diss 37: 1-7.

Roughton FJW (1936) The thermo-chemistry of the oxygen-haemoglobin reaction II. Comparison of the heat as measured directly on purified hemoglobin with that calculated directly by the vant' Hoff isochor. Biochem J 30: 2117-2133.

Scalonia A, Ferrantia P, De Simoned G, Mamonea G, Sannoloa N, Malornia A (1999) Probing the reactivity of nucleophile residues in human 2,3-diphosphoglycerate/deoxy-hemoglobin complex by a specific chemical modifications. FEBS Lett 452: 190-194.

Shmukler BE, Brugnara C, Alper SL (2000) Structure and genetic polymorphism of the mouse KCC1 gene. Biochim Biophys Acta 1492: 353-361.

Stathopulos PB (2003) Taking the good out of the bad: lentiviral-based gene therapy of the hemoglobinopathies. Biotechnology Adv 21: 513-526.

Steiper ME, Wolfe ND, Karesh WB, Kilbourn AM, Bosi EJ, Ruvolo M (2006) The phylogenetic and evolutionary history of a novel alpha-globin-type gene in orangutans (Pongo pygmaeus). Infection Genet Evol 6: 277-286.

Tyuma I, Imai K, Shimizu K (1973) Analysis of oxygen equilibrium of hemoglobin and control mechanism of organic phosphates. Biochemistry 12: 1491-1498.

Tyuma I, Kamigawara Y, Imai K (1973) pH Dependence of the shape of the hemoglobin-oxygen equilibrium curve. Biochim Biophys Acta 310: 317-320.

Wyman J (1948) Heme proteins. Adv Protein Chem 4: 407531.

Yamamoto Y, Nagaoka T (1998) A ${ }^{1} \mathrm{H}$ NMR comparative study of human adult and fetal hemoglobin's. FEBS Lett 424: 169-172. 